\title{
Gerenciamento clínico de eventos tromboembólicos provenientes de complicações da COVID-19: revisão integrativa
}

\author{
Clinical management of thromboembolic events arising from complications of COVID-19: \\ integrative review \\ Manejo clínico de eventos tromboembólicos derivados de complicaciones del COVID-19: revisión \\ integrativa
}

Recebido: 24/09/2021 | Revisado: 30/09/2021 | Aceito: 04/10/2021 | Publicado: 09/10/2021

\author{
Laura Dayane Gois Bispo \\ ORCID: https://orcid.org/0000-0003-1628-520X \\ Universidade Federal de Sergipe, Brasil \\ E-mail: lauradayane2010@ hotmail.com \\ Natan Martins Machado \\ ORCID: https://orcid.org/0000-0002-6745-1231 \\ Universidade Federal de Sergipe, Brasil \\ E-mail: natan08martins@ hotmail.com \\ Caio Lopes Pinheiro de Paula \\ ORCID: https://orcid.org/0000-0003-0848-2473 \\ Hospital de Urgências de Sergipe, Brasil \\ E-mail: caiolopespp@gmail.com \\ Jussiely Cunha Oliveira \\ ORCID: https://orcid.org/0000-0001-6782-8994 \\ Universidade Federal de Sergipe, Brasil \\ E-mail: jussiely@hotmail.com \\ Ingrid Melo Santos \\ ORCID: https://orcid.org/0000-0001-7831-1325 \\ Universidade Tiradentes, Brasil \\ E-mail: ingridmeelo@ hotmail.com \\ Fernanda Gomes de Magalhães Soares Pinheiro \\ ORCID: https://orcid.org/0000-0002-5258-4752 \\ Universidade Federal de Sergipe, Brasil \\ E-mail: fernandagmsoares@gmail.com
}

\begin{abstract}
Resumo
Objetivo: identificar de que forma ocorre o gerenciamento clínico de pacientes infectados por COVID-19 que apresentam tromboembolismo pulmonar e/ou tromboembolismo venoso. Método: Trata-se de uma revisão integrativa, realizada através da busca de artigos nas bases de dados BVS, LILACS, MEDLINE, CINAHL E SCOPUS, com delimitação temporal de novembro de 2019 a março de 2021. Resultados: Foram incluídos 24 artigos. O diagnóstico ocorre através do D-dímero e fibrinogênio. Para a terapia medicamentosa, administra-se a Heparina de baixo peso molecular (HBPM), sendo $30 \mathrm{mg} / 12 \mathrm{~h}$ em $12 \mathrm{~h}$ para a anticogulação profilática, doses escalonadas de HBPM com enoxaparina, $0,5 \mathrm{mg} / \mathrm{kg}$ duas vezes por dia para a anticoagulação terapêutica e rivoraxabana 10mg/dia para a anticoagulação pós alta. Conclusão: O gerenciamento clínico de TEP e TEV ocorre através do aumento do D-dímero ou do fibrinogênio, com o TEP confirmado através da angiografia pulmonar. Para a anticoagulação, utiliza-se a HBPM, maiormente.
\end{abstract}

Palavras-chave: Tromboembolia venosa; Embolia pulmonar; COVID-19; Gerenciamento clínico.

\begin{abstract}
Objective: to identify how the clinical management of patients infected with COVID-19 who present pulmonary thromboembolism and/or venous thromboembolism occurs. Method: This is an integrative review, carried out by searching for articles in the VHL, LILACS, MEDLINE, CINAHL AND SCOPUS databases, with a temporal delimitation from November 2019 to March 2021. Results: 24 articles were included. Diagnosis occurs through D-dimer and fibrinogen. For drug therapy, low molecular weight heparin (LMWH) is administered, $30 \mathrm{mg} / 12 \mathrm{~h}$ in $12 \mathrm{~h}$ for prophylactic anticoagulation, staggered doses of LMWH with enoxaparin, $0.5 \mathrm{mg} / \mathrm{kg}$ twice a day for anticoagulation therapy and rivoxaban $10 \mathrm{mg} /$ day for post-discharge anticoagulation. Conclusion: Clinical management of TEP and VTE occurs through increased D-dimer or fibrinogen, with TEP confirmed by pulmonary angiography. For anticoagulation, LMWH is mostly used.
\end{abstract}

Keywords: Venous thromboembolism; Pulmonary embolism; COVID-19; Clinical management. 


\begin{abstract}
Resumen
Objetivo: identificar cómo se produce el manejo clínico de los pacientes infectados por COVID-19 que presentan tromboembolismo pulmonar y / o tromboembolismo venoso. Método: Se trata de una revisión integradora, realizada mediante la búsqueda de artículos en las bases de datos BVS, LILACS, MEDLINE, CINAHL Y SCOPUS, con una delimitación temporal de noviembre de 2019 a marzo de 2021. Resultados: Se incluyeron 24 artículos. El diagnóstico se produce a través del dímero D y el fibrinógeno. Para la farmacoterapia se administra heparina de bajo peso molecular (HBPM), $30 \mathrm{mg} / 12 \mathrm{~h}$ en $12 \mathrm{~h}$ para la anticoagulación profiláctica, dosis escalonadas de HBPM con enoxaparina, 0,5 $\mathrm{mg} / \mathrm{kg}$ dos veces al día para la anticoagulación y rivoxabán $10 \mathrm{mg} /$ día para el postratamiento. anticoagulación. Conclusión: El tratamiento clínico de la TEP y la TEV se produce a través del aumento del dímero D o del fibrinógeno, y la TEP se confirma mediante una angiografía pulmonar. Para la anticoagulación, se usa principalmente HBPM.

Palabras clave: Tromboembolismo venoso; Embolia pulmonar; COVID-19; Gestión clínica.
\end{abstract}

\title{
1. Introdução
}

O desenvolvimento de coagulopatias decorrente da infecção pelo Coronavirus Disease 2019 (COVID-19) apresenta uma incidência de $20 \%$ nos pacientes graves. Esta incidência é mais significativa, visto que cerca de $100 \%$ apresentam anormalidades de coagulação, subsequente ao aumento das citocinas inflamatórias (IL-1 $\beta$, IL-6 e IL-10, principalmente) devido à infecção viral, o que provoca uma síndrome tromboinflamatória endotelial e propicia à coagulopatia microvascular (Zhai, et al., 2020; Ciceri, et al., 2020; Yang, et al., 2020).

Nesse contexto, a trombocitopenia é um achado comum, o que triplica o risco de gravidade e aumenta a mortalidade em 45\%, quando comparada às demais causas de mortalidade hospitalar (Lippi, et al., 2020; Liao, et al., 2020). O aumento do Ddímero e fibrinogênio são associados a evolução das coagulopatias, o que pode ser piorado pela degradação da fibrina, decorrentes desse processo inflamatório viral. Tais produtos parecem repercutir de uma forma subjetiva nos pacientes, de modo a tornar o gerenciamento clínico controverso, visto que os aspectos clínicos são individuais e, mesmo que o processo de saúde-doença seja semelhante, ainda falta evidência científica, sólida, para definição de condutas clínicas (Rossi, et al., 2020; Marietta, et al., 2020).

O gerenciamento clínico é todo processo de tratamento da doença e deve ponderar a efetividade das terapias disponíveis e os resultados dos pacientes (Brasil, 2021). As coagulopatias por COVID-19 são gerenciadas entre anticoagulação profilática, terapêutica (diagnóstico confirmado) e pós alta hospitalar. Porém, diferentes questionamentos sobre a eficácia do regime medicamentoso são presentes na literatura, o que geram desafios na prática clínica (Dobesh, et al., 2020). Tal desafio é visto pelo aumento de casos de tromboembolismo venoso (TEV) e tromboembolismo pulmonar (TEP), que ocorrem em $25 \%$ e $30 \%$ dos pacientes, respectivamente, nos quais ambos são associados à piores prognósticos dos pacientes (Middeldorp, et al., 2020; Cui, et al., 2020).

No tocante ao gerenciamento clínico da TEV, a anticoagulação profilática é realizada por meio da administração da enoxaparina (Cui, et al., 2020; Bikdeli, et al., 2020). Para a realização da anticoagulação terapêutica, utiliza-se a heparina de baixo peso molecular (HPBM) e, para o seguimento pós alta hospitalar, é recomendado, preferencialmente, o uso dos anticoagulantes orais, com atenção para o risco de sangramento (Aryal, et al., 2020; Tang, et al., 2020; Liao, et al. 2020). Tratamento semelhante ocorre no TEP (Haque, et al., 2020).

É válido ressaltar que ainda não há consenso e evidências científicas, com estudos robustos, sobre a terapia medicamentosa para TEP e TEV, o que justifica a necessidade de estudos sobre o gerenciamento clínico desses eventos tromboembólicos, desde a prevenção das coagulopatias até o manejo pós alta, sendo importante conciliar regimes terapêuticos e medicamentosos, baseados em diferentes grupos de pacientes, incluindo o custo-benefício (Vernuccio, et al., 2020; Rosovsky, et al., 2020; Marietta, et al., 2020).

Dada a divergência na conduta clínica das coagulopatias por COVID-19, destaca-se como importante a análise do gerenciamento clínico de TEP e TEV, desde que provenientes de complicações da COVID-19. Logo, esta investigação 
justifica-se devido à necessidade de orientação no raciocínio no que concerne ao manejo clínico, baseados nas evidências disponíveis, tendo como objetivo identificar de que forma ocorre o gerenciamento clínico de pacientes infectados por COVID19 que apresentam tromboembolismo pulmonar e/ou tromboembolismo venoso.

\section{Metodologia}

Revisão integrativa, constituída das etapas: identificação da questão norteadora, estabelecimento de critérios de inclusão e de exclusão; categorização dos artigos (extração, organização e sumarização das informações); avaliação dos estudos incluidos; interpretação dos resultados e a presentação da revisão, ambas sugeridas pelo estudo de Mendes e colaboradores (2008).

Utilizando a estratégia PIC (população, fenômeno, contexto), foi elaborada a questão: Como é feito o gerenciamento clínico e terapêtico de pacientes infectados por COVID-19 que apresentam TEP ou TEV? Os critérios de inclusão foram estudo primários que incluíram pacientes maiores de 18 anos, com eventos tromboembólicos, TEV e/ou TEP, pós infecção por coronavírus, publicados entre novembro de 2019, início da pandemia até a março de 2021. Foram excluídos artigos; títulos e/ou resumos que não se enquadravam na temática e/ou não respondiam à pergunta.

A coleta de dados foi realizada no portal de periódicos da Coordenação de Aperfeiçoamento de Pessoal de Nível Superior (CAPES), nas bases de dados: Biblioteca Virtual em Saúde (BVS), Literatura Latino-Americana e do Caribe em Ciências da Saúde (LILACS), Medical Literature Analysis and Retrieval System Online (MEDLINE), Cumulative Index to Nursing and Allied Health Literature (CINAHL) e SCOPUS. Os descritores utilizados, todos controlados, se encontram no Banco de Descritores em Ciências da Saúde (Gerenciamento Clínico; Terapêutica; Conduta do Tratamento Medicamentoso; Infecções por Coronavirus; Surto pelo Coronavírus 2019-nCoV; COVID-19; Tromboembolia Venosa e Embolia Pulmonar) e no Medical Subject Headings (Disease Management; Therapeutic; COVID-19 drug treatment; COVID-19; SARS-CoV-2; Thromboembolism; Venous Thromboembolism e Pulmonary Embolism). Foram empregados os operadores booleanos "OR" e “AND”. Contudo, foram utilizadas diferentes estratégias de busca, ao considerar que as bases de dados possuem peculiaridades para a construção das estratégias (Quadro 1).

Quadro 1- Estratégia de busca nas bases de dados. Lagarto (SE),2021.

\begin{tabular}{|c|l|}
\hline $\begin{array}{c}\text { Base de } \\
\text { Dados }\end{array}$ & \multicolumn{1}{c|}{ Estratégia de busca utilizada } \\
\hline BVS & $\begin{array}{l}\text { (Gerenciamento Clínico) OR (Terapêutica) OR (Conduta do Tratamento Medicamentoso) AND (Infecções por Coronavirus) OR } \\
\text { (Surto pelo Coronavírus 2019-nCoV) OR (COVID-19) AND (Tromboembolia Venosa) OR (Embolia Pulmonar) }\end{array}$ \\
\hline LILACS & $\begin{array}{l}\text { (Gerenciamento Clínico) OR (Terapêutica) OR (Conduta do Tratamento Medicamentoso) AND (Infecções por Coronavirus) OR } \\
\text { (Surto pelo Coronavírus 2019-nCoV) OR (COVID-19) AND (Tromboembolia Venosa) OR (Embolia Pulmonar) }\end{array}$ \\
\hline MEDLINE & $\begin{array}{l}\text { ("Disease Management"[Mesh] OR "Therapeutics"[Mesh]) AND ("COVID-19 drug treatment" [Supplementary Concept] OR } \\
\text { "COVID-19"[Mesh] OR "SARS-CoV-2"[Mesh]) AND ("Thromboembolism"[Mesh] OR "Venous Thromboembolism"[Mesh] } \\
\text { OR "Pulmonary Embolism"[Mesh]) }\end{array}$ \\
\hline SCOPUS & $\begin{array}{l}\text { (Disease Management) AND (Therapeutic) AND (COVID-19) AND (Venous Thromboembolism) OR (Pulmonary Embolism) } \\
\text { (Thromboembolism) OR (Venous Thromboembolism) OR (Pulmonary Embolism) }\end{array}$ \\
\hline
\end{tabular}

Fonte: Autores (2021) 
Inicialmente, foram selecionados 03 artigos na LILACS, 181 na MEDLINE, 53 no SCOPUS, 39 na BVS e 998 na CINAHL, resultando em 1274 artigos. Após a aplicação dos critérios de inclusão e exclusão, obteve-se 94 artigos, os quais foram lidos e analisados na íntegra. Após a identificação, os artigos foram submetidos a um processo de triagem, por meio de análise da temática abordada, que incluía leitura do título, resumo e análise segundo critérios de inclusão e exclusão. Nesse processo, os artigos duplicados entre bases de dados e aqueles repetidos entre os selecionados foram identificados, tendo sido realizada a eleição definitiva das referências elegíveis para leitura na íntegra. Utilizou-se como guia o instrumento Preferred Reporting Items for Systematic Review and Meta-Analyses (PRISMA) para auxiliar no processo de identificação, triagem, elegibilidade e inclusão dos estudos (Galvão, et al., 2015), conforme representado na Figura 1.

Figura 1 - Processo de busca e seleção dos artigos por meio de cruzamentos dos termos Medical Subject Headings (MeSH) e Descritores em Ciências da Saúde (DeCS), via bases de dados e biblioteca virtual.

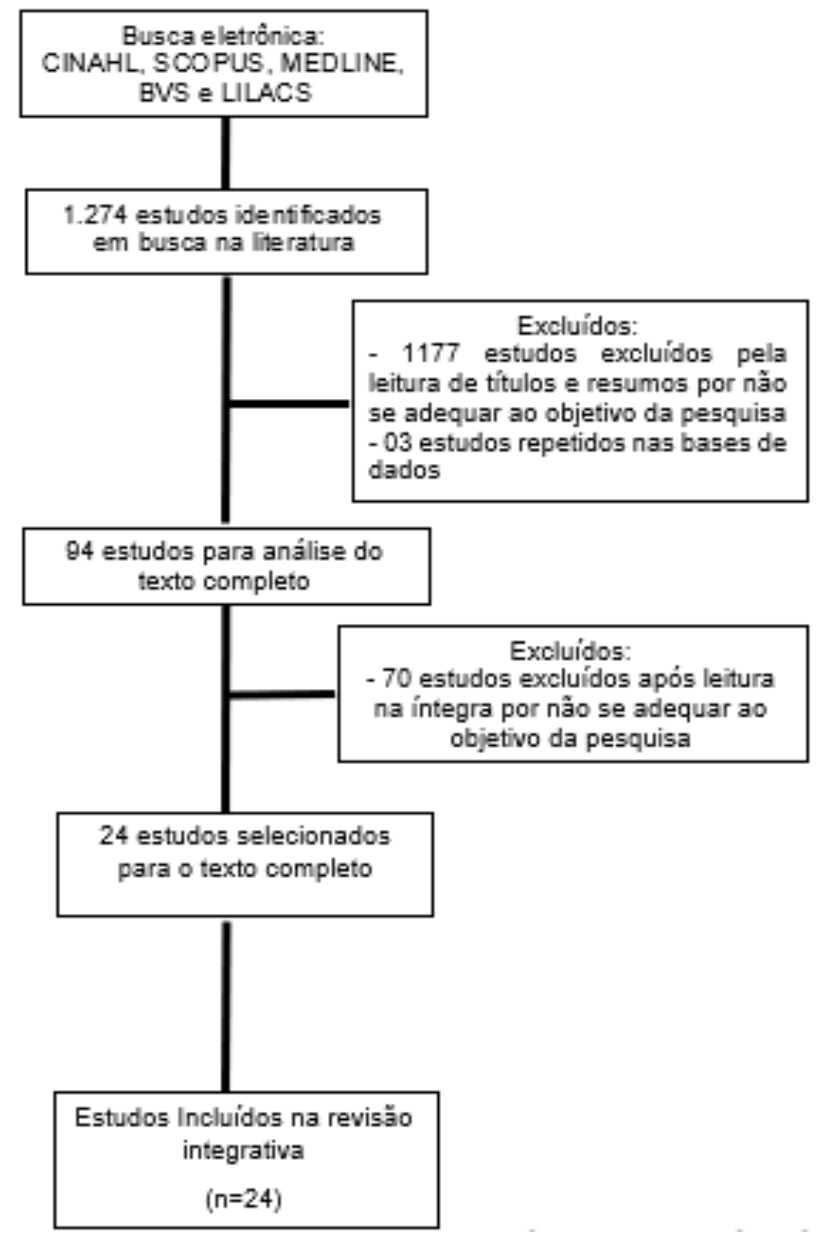

BVS: Biblioteca Virtual em Saúde; CINAHL: Cumulative Index to Nursing and Allied Health Literature; MEDLINE®: Medical Literature Analysis and Retrieval System Online; LILACS: Literatura Latino-Americana e do Caribe em Ciências da Saúde.

Fonte: Autores (2021).

De modo a assegurar a qualidade dessas etapas e evitar vieses de seleção, adotou-se a seleção duplo-independente. Em situações de divergência, um terceiro avaliador foi consultado. Para extração dos dados, utilizou-se um instrumento elaborado pelos pesquisadores, contendo as variáveis: autoria; ano; país; objetivo; população de estudo; método; tipo de intervenção e duração da intervenção (quando aplicável); resultados; conclusões e título.

A análise procedeu com a caracterização bibliométrica, sendo organizado o banco de dados do estudo em planilha do programa Microsoft Office Excel® versão 2019. Os revisores adotaram a estratégia visual verde- incluídos; amarelos- releitura 
e vermelho-excluídos. Posteriormente, realizou-se uma síntese qualitativa dos estudos incluídos, de forma descritiva, sendo possível organizar os dados em uma estrutura lógica: simplificar, sumarizar e comparar sistematicamente os resultados oriundos dos estudos primários sobre a questão em estudo, de modo a facilitar o processo de análise interpretativa, com posterior discussão dos achados, em conformidade com a literatura científica.

Para o nível de evidência, foi considerada a classificação proposta por Melnky e colaboradores (2011): nível IEvidências oriundas de revisão sistemática ou meta-análise de todos os ensaios clínicos relevantes randomizados, controlados ou provenientes de diretrizes clínicas baseadas em revisões sistemáticas de ensaios clínicos randomizados controlados; nível II-Evidências derivadas de pelo menos um ensaio clínico randomizado controlado bem delineado; nível III-Evidências obtidas de ensaios clínicos bem delineados sem randomização; nível IV-Evidências provenientes de estudos de coorte e de casocontrole bem delineados; nível V-Evidências originárias de revisão sistemática de estudos descritivos e qualitativos; nível VIEvidências derivadas de um único estudo descritivo ou qualitativo; nível VII-Evidências oriundas de opinião de autoridades e/ou relatório de comitês de especialistas.

\section{Resultados}

Foram incluídos 24 artigos, publicados em 11 países: 2 na China (8\%), 2 na Grécia (8\%), 4 na Itália (17\%), 6 nos Estados Unidos (25\%), 1 no Irã (4\%), 1 nos Emirados Árabes Unidos (4\%), 1 na Turquia (4\%), 2 na Bélgica (8\%), 1 na França (4\%), 3 na Espanha (13\%) e 1 no Reino Unido (4\%), os quais todos foram publicados na língua inglesa. A maioria dos estudos foi publicado no ano de 2020, totalizando 18 publicações, correspondendo a 75\% da amostra e no ano de 2021, com 6 publicações, colaborando com 25\% da amostra. Quanto à base de dados, 14 artigos foram publicados na MEDLINE (58\%), 4 na SCOPUS (17\%) e 6 na CINAHL (25\%).

Ao analisar o delineamento das pesquisas, foram encontrados 14 estudos de coorte (58\%), 1 série de casos (4\%), 2 revisões sistemáticas (8\%), 5 estudos retrospectivos (21\%), 1 estudo multicêntrico (4\%) e 1 estudo monocêntrico (4\%). Os estudos abrangeram os níveis IV, V e VI de evidência, com 17 no nível IV (71\%), 2 no nível V (8\%) e 5 no nível VI (21\%). Devido a presente revisão integrativa se tratar de duas patologias (Tromboembolismo Venoso e Tromboembolismo Pulmonar), o quadro (https://drive.google.com/file/d/1j3v-JTgfyYBA0sKKdrDwMy687HWE8Csd/view?usp=sharing) está organizado da seguinte forma: artigos referentes às duas patologias; apenas ao Tromboembolismo Venoso e apenas ao Tromboembolismo Pulmonar.

\section{Discussão}

\section{Gerenciamento Clínico do Tromboembolismo Venoso e Pulmonar}

Em relação ao gerenciamento clínico, 11 artigos (46\%) abordavam sobre TEP e TEV, 5 artigos (21\%) apenas sobre TEV e 08 artigos (33\%) apenas sobre TEP. Para a presente discussão, o gerenciamento clínico será dividido em: epidemiologia, fatores de risco, manifestações clínicas, diagnóstico, tratamento e manejo pós alta. Houve uma predominância de estudos com ênfase no tratamento (71\%). Porém, os demais atributos do gerenciamento clínico conseguiram ser sanados.

Quanto à epidemiologia, 08 estudos (33\%) abordaram o perfil epidemiológico dos pacientes que tiverem TEP ou TEV. A incidência de TEV variou entre $0,5 \%$ a 42,2\%, com a prevalência de, aproximadamente, $20 \%$ para TEV (Rashisi, $e t$ al., 2021; Trigonis, et al., 2020; Minno, et al., 2020). A incidência de TEP foi 6,4\% (Mestre-Gomez, et al., 2020). O tamanho amostral dos estudos teve uma diferença significativa, abrangendo entre 21 e 4389 pacientes (Nadkarni, et al., 2020; GarciaOlivé, et al., 2020). Os estudos incluídos, em média, apresentam uma incidência divergente do previsto na literatura. Nopp et al. (2020), por exemplo, avaliaram a incidência global de tromboembolismo em 22,7\% para TEV e 14,1\% para TEP. 
A idade variou de 27 a 96 anos, com predominância de pacientes idosos (65-70 anos) (42\%) e do sexo masculino (67\%). Houve uma prevalência de eventos tromboembólicos associados ao Índice de Massa Corpórea (IMC), dislipidemias e Diabetes Mellitus (Minno, et al., 2020; Valle, et al., 2020). Tais achados já eram esperados na literatura, devido as patologias de caráter inflamatório, como Diabetes Mellitus e obesidade, aumentarem o tempo de hospitalização e a mortalidade (Marinho, et al., 2021).

Dos estudos incluídos, dois (07\%) abordaram os fatores de risco para eventos tromboembólicos, nos casos de COVID-19. Os principais fatores de risco foram maior nível sérico de Interleucina-6, taquipneia e diminuição da Saturação Periférica de Oxigênio $\left(\mathrm{SpO}_{2}\right)$ e outros fatores, relacionados à embolia pulmonar: acidente vascular cerebral prévio, fibrilação atrial, dor torácica ou dispneia (Fauvel, et al., 2020; Garcia-Ortega, et al., 2020).

Quanto às manifestações clínicas, os achados mais comuns foram tosse e dispneia (Garcia-Ortega, et al., 2020). Outros estudos descreveram que os pacientes, com eventos tromboembólicos, apresentavam febre, dispneia, palpitação, fadiga, distúrbios musculoesqueléticos e pneumonia (Long, et al., 2020; Rashidi, et al., 2021; Bozzani, et al., 2020). Tais achados também foram apresentados por Eljilany e colaboradores, que incluíram que o tratamento, oxigenioterapia ou intubação podem dificultar a suspeita e diagnóstico de tromboembolismo (Eljilany, et al., 2020).

No que concerne ao diagnóstico do TEV e TEP, dez estudos (42\%) recomendaram a avaliação do D-dímero, fibrinogênio, tempo de protrombina e tromboplastina parcial ativada, além dos exames de imagem, tomografia computadorizada e angiograma pulmonar por tomografia computadorizada, este padrão ouro para o diagnóstico da embolia pulmonar (Ooi, et al., 2020; Gomes, et al., 2006). O valor do D-dímero tem aumento típico nos 07 primeiros dias para o diagnóstico de TEV, devido à COVID-19 (Creel-Bulos, et al., 2020). Os mesmos autores identificaram que o D-dímero, nos primeiros 7 dias de hospitalização, variou entre 6450 vs.1596, nos pacientes com COVID-19, que desenvolveram TEV em comparação com casos de não TEV. Assim, um dos critérios para diagnóstico de TEV associado às complicações da COVID19 é o D-dímero $>2.000 \mathrm{ng} / \mathrm{mL} / \mathrm{d}$ ou uma taxa de alteração superior a $150 \mathrm{ng} / \mathrm{mL} / \mathrm{d}$ em relação ao último valor coletado, no período de 24 horas até o décimo dia de hospitalização. Caso o D-dímero esteja >2000ng/mL, Trigonis et al. (2020) recomendam, também, a avaliação por meio de exames de imagem (ultrassom).

Ademais, a dosagem do D-dímero também é recomendada para o diagnóstico de TEP, quando complicação da COVID-19 (Fauvel, et al., 2020; Garcia-Olivé, et al., 2020; Valle, et al., 2020). Porém, Mestre-Gomez et al. (2020) apontam divergências quanto ao valor, de forma a diagnosticar o TEP a partir do D-dímero> $5000 \mu \mathrm{g} / \mathrm{dl}$. Em relação ao risco de desenvolver TEP, evidencia-se o aumento do D-dímero no $3^{\circ}, 6^{\circ}$ e $9^{\circ}$ dia (Garcia-Olivé, et al., 2020). Tanto as lesões pulmonares graves na Tomografia, como a Fração inspirada de Oxigênio (FiO2) alta também foram associados ao maior risco de embolia pulmonar (Fauvel, et al., 2020). Essa estratégia e esses critérios para estabelecimento diagnóstico também são considerados por Elijiany et al. (2020).

Os estudos também citaram o aumento do fibrinogênio para o diagnóstico de TEP e TEV, porém não citam valores laboratoriais (Trigonis, et al., 2020; Long, et al., 2020). A literatura evidencia o fibrinogênio < $2 \mathrm{~g} / \mathrm{L}$ para fim diagnóstico de eventos tromboembólicos, associados à COVID-19 (Eljilany, et al., 2020). Além disso, o aumento do fibrinogênio, do tempo de protrombina e do tempo de tromboplastina parcial ativada, podem ser utilizados como indicadores de progressão do TEV e TEP (Long, et al., 2020).

Além de exames laboratoriais e de imagem, a aplicação de escores clínicos, com finalidade de estimar a probabilidade para o Tromboembolismo Pulmonar (TEP), são úteis na prática clínica (Robert-Ebadi, et al., 2017). Fu et al. (2020) utilizaram o Escore de Genebra, no auxílio diagnóstico de TEP e concluíram que este escore, associado à dosagem de D-dímero, melhoram a precisão diagnóstica. O escore de Genebra consiste na análise de oito variáveis para predizer o diagnóstico de embolia, sendo: idade > 65 anos; TEP ou TEV prévio; história de cirurgia ou fratura dentro de 1 mês; condição maligna ativa 
(sólida ou hematológica), mesmo que curada; dor unilateral nos membros inferiores; hemoptise; Frequência cardíaca > 95bpm e dor ou edema na palpação dos membros inferiores (Klok, et al., 2008). As variáveis possuem uma pontuação entre um e três pontos e pontuação menor ou igual à dois, é classificada como embolia pulmonar improvável e maior ou igual a três como provável embolia pulmonar (Fu, et al., 2020).

Para uma melhor precisão diagnóstica de TEP e TEV, há necessidade da disponibilização de exames de imagens tanto na rotina, como nos protocolos de atendimento. Lodigiani et al. (2020) sugerem que a subnotificação de TEP e TEV pode estar associada à menor disponibilidade de exames de imagens mais específicos, como a angiografia pulmonar computadorizada. Nesse sentido, Valle et al. (2020) indicam que a angiografia pulmonar foi eficaz no diagnóstico de 57\% casos de TEP, com extensão pulmonar diretamente proporcional ao aumento do D-dímero. Tais fatos são assertivos com a literatura, que recomenda a avaliação por exames de imagem, principalmente se o D-dímero crescer exponencialmente (Parks, et al., 2020). Tanto a angiografia pulmonar como a venografia por tomografia computadorizada constituem o protocolo padrão para a investigação de embolia pulmonar (Gomes, et al., 2006; Fu, et al., 2020).

Quanto ao tratamento farmacológico, as principais medicações são heparina de baixo peso molecular, heparina não fracionada, anticoagulantes orais, agentes antiplaquetários, inibidores do fator XII e drogas trombolíticas (Manolis, et al., 2021). A heparina de baixo peso molecular é a mais utilizada, tendo em vista seus efeitos anti-inflamatórios, além do anticoagulante (Manolis, et al., 2021; Lodigiani, et al., 2020; Trigonis, et al., 2020; Bozzani, et al., 2020). Smith et al. (2020) acrescentam a administração do Fondaparinux $2,5 \mathrm{mg} /$ subcutânea para pacientes com histórico de trombocitopenia induzida por Heparina.

No que concerne à anticoagulação profilática, houve uma variedade de recomendações entre os artigos incluídos. Trigonis et al. (2020) recomendam a administração da heparina de baixo peso molecular (HBPM), $30 \mathrm{mg} / 12 \mathrm{~h}$ em $12 \mathrm{~h}$. Garcia Ortega et al. (2020), no entanto, recomendam a enoxaparina $40 \mathrm{mg}$ ou $60 \mathrm{mg} / \mathrm{dia}$. Doses semelhantes são recomendadas nos demais estudos incluídos (Rashidi, et al., 2021; Canoglu, et al., 2020; Atallah, et al., 2021; Li, et al., 2020). Também, é indicada a utilização da enoxaparina $0,5 \mathrm{mg} / \mathrm{kg}$ 2x/dia, Heparina não fracionada 4000 a $10.000 \mathrm{UI}$, subcutânea-3x/dia ou a administração de Dalteparina 5000UI subcutânea, 3x/dia, em casos de insuficiência renal (Canoglu, et al., 2020; Manolis, et al., 2021; billet, et al., 2020).

Smith et al. (2020) defendem a anticoagulação profilática, a ser administrada de diferentes maneiras, em níveis: Nível I- anticoagulação de baixa intensidade (HBPM em doses profiláticas padrão- Enoxaparina subcutânea 30mg 1x/dia); Nível IIanticoagulação de intensidade intermediária (Enoxaparina $40 \mathrm{mg}$ ou 0,5mg/kg subcutânea $12 \mathrm{em} 12$ h, a última opção em casos de obesidade) e Nível III- pacientes com eventos tromboembólicos confirmado ou fortemente suspeito de TEV (critério: D-dímero $>6 \mu \mathrm{g} / \mathrm{dl}$ ): dosagem terapêutica de HBPM, como Enoxaparina $1 \mathrm{mg} / \mathrm{kg}$ subcutânea a cada 12 horas (creatinina $>30$ $\mathrm{mL} / \mathrm{min}$ ), $1 \mathrm{mg} / \mathrm{kg}$ subcutânea $1 \mathrm{x} / \mathrm{dia}$ (creatinina $<30 \mathrm{~mL} / \mathrm{min}$ ) ou Heparina não fracionada EV titulado, conforme protocolo institucional. Em contrapartida, Lodigiani et al. (2020) são desfavoráveis à tromboprofilaxia como rotina hospitalar, sob o argumento de não ser possível medir os resultados e reais benefícios.

Para a anticoagulação terapêutica, os estudos recomendam a utilização da Apixabana $5 \mathrm{mg}$ ou Rivorababana $10 \mathrm{mg}$, HBPM $1 \mathrm{mg} / \mathrm{kg}$ 2x/dia ou doses escalonadas de HBPM com enoxaparina, $0,5 \mathrm{mg} / \mathrm{kg}$ duas vezes por dia, embora pacientes com comorbidade precisem de ajustes da medicação a ser utilizada e/ou da dosagem, a citar os pacientes com insuficiência renal, nos quais são preferíveis, a heparina não fracionada (Billet, et al., 2020; Poulakou, et al., 2021; Canoglu, et al., 2020; Li, et al., 2020; Bozzani, et al., 2020).

Os regimes de anticoagulação profilática e terapêutica são questionados, devido ao risco de sangramento. Houve sangramento pós anticoagulação nos estudos de Lodigiani et al. (2020), Rashidi et al. (2021), Nadkarni et al. (2020), Atallah et 
al. (2021) e Li et al. (2020), enquanto houve ausência de sangramento nos estudos de Manolis et al. (2021) e Rentsch et al. (2021). Pavoni et al. (2020) concordam com tais regimes, visto que o risco tromboembólico é maior que o risco hemorrágico.

Quanto à anticoagulação pós alta, foi recomendada a profilaxia por 30 dias a 45 dias, em média, o que confirma com o preconizado em literatura, sendo os anticoagulantes orais os mais utilizados nessa etapa (Nadkarni, et al., 2020; Spyropoulos, et al., 2020). Bozzani et al. (2020) recomendam a administração de aspirina de baixa dose ( $81 \mathrm{mg} / 2 \mathrm{x} / \mathrm{dia})$ por 4 semanas após a alta, quando o paciente é considerado baixo risco. A literatura, ademais, sugere utilizar a rivoraxabana 10mg/dia, via oral (Rossi, et al., 2020).

\section{Limitações do estudo}

Diante da pandemia e da necessidade de publicações rápidas sobre o assunto, estima-se que estudos com desenhos metodológicos mais robustos e de maiores validades, como ensaios clínicos randomizados, estudos multicêntricos e revisões sistemáticas, podem ainda estar em desenvolvimento, de modo a interferir nos níveis de evidências e, consequentemente, limitando as respostas de muitas pesquisas. Além disso, o gerenciamento clínico baseado em protocolos sem validação pode comprometer a eficácia do regime terapêutico proposto nesta revisão integrativa.

\section{Conclusão}

O gerenciamento clínico de pacientes infectados por COVID-19 que apresentam tromboembolismo pulmonar e/ou

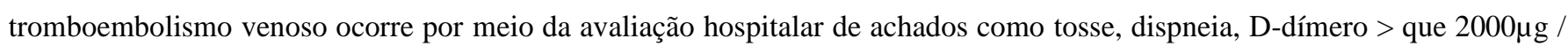
$\mathrm{dl}$ ou com $>150 \mu \mathrm{g} / \mathrm{dl}$ de alteração entre o último valor coletado ou aumento do fibrinogênio para >2g/L, com confirmação por angiografia pulmonar, o último como preferencial para o diagnóstico do Tromboembolismo Pulmonar. Para o tratamento, divide-se entre a anticoagulação profilática e terapêutica, com a utilização da Heparina de Baixo Peso Molecular para ambas, maiormente. Aconselha-se a anticoagulação pós alta, quando não contraindicada, por até 45 dias.

A produção cientifica e busca pelas melhores evidências durante a pandemia sobre questões referentes à ação inflamatória sistêmica e trombogênica na COVID-19 tem sido amplamente realizada. Contudo, alguns tipos de estudos requerem cuidados na interpretação dos resultados. Assim, são esperadas pesquisas clínicas, ensaios clínicos e revisões sistemáticas, bem conduzidas, que possam subsidiar o raciocínio clínico quanto às melhores práticas terapêuticas nos pacientes com eventos tromboembólicos provenientes da COVID-19. Ainda, são esperados que futuros trabalhos busquem a validação de protocolos de gerenciamento clínico de eventos tromboembólicos preexistentes, de forma a contribuir com a segurança da prática clínica.

\section{Referências}

Aryal, M. R., Gosain, R., Donato, A., Pathak, R., Bhatt, V. R., KateL, A. \& Kouides, P. (2020). Venous Thromboembolism in COVID-19: Towards an Ideal Approach to Thromboprophylaxis, Screening, and Treatment. Current Cardiology Report, 22(52), 01-05.

Atallah, B., Sadik, G. B., Salem, N., Nekidy, W. S. E., Almahmeed, W., Park, W. M. .. Mallat, J. (2021). The impact of protocol-based high-intensity pharmacological thromboprophylaxis on thrombotic events in critically ill COVID-19 patients. Anaesthesia, 76, 327-335.

Bikdeli, M. S. D., Madavan, M. V., Jimenez, D., Chuich, T., Dreyfus, I., Driggin, E, ... Lip, G. Y. H. (2020). COVID-19 e Trombotic ouDoença tromboembólica: Implicaçõespara prevenção, terapia antitrombótica,e Follow-Up. Journal of the American college of cardiology, 75(23), $2950-2973$.

Billet, H. H., Reyes-Gil, M., Szymanski, J., Ikemura, K., Stahl, L.R. \& Lo, Y. (2020). Anticoagulation in COVID-19: Effect of Enoxaparin, Heparin, and Apixaban on Mortality. Thrombosis and Haemostasis, 120(12), 1691-1699.

BRASIL- Biblioteca Virtual em Saúde (2021). Gerenciamento Clínico. Pesquisa | Portal Regional da BVS (bvsalud.org).

Bozzani, A., Arici, V., Tavazzi, G., Franciscone, M. M., Danesino, V., Rota, M. ... Ragni, F. (2020). Acute arterial and deep venous thromboembolism in COVID-19 patients: Risk factors and personalized therapy. Surgery, 98, 987-992. 
Canoglu, K. \& Saylan, B. (2020). Therapeutic dosing of low-molecular-weight heparin may decrease mortality in patients with severe COVID-19 infection. ANN SAUDI MED, 462-468.

Ciceri, F., Beretta, L., Scandroglio, A.M., Colombo, S., Landoni, G., Ruggeri, A., Peccatori, J., D’Angelo, A., Cobelli, F., Rovere-Querini, P., Tresoldi, M, Dagna, L. \& Zangrillo, A. (2020). Microvascular COVID-19 síndrome tromboinflamatória obstrutiva dos vasos pulmonares (MicroCLOTS): uma hipótese de trabalho da síndrome da angústia respiratória aguda atípica. Crit Care Resusc., 22(02), 95-77.

Creel-Bulos, C., Lui, M., Auld, S. C., Gaddh, M., Kemptom, C. L., Sharipfour, M. ... Rangaraju, S. (2020). Trends and diagnostic value of D-dimer levels in patients hospitalized with coronavirus disease 2019. Medicine, 99(46), 01-08.

Cui, S., CheN, S., Li, X., liu, S. \& Wang, F. (2020) Prevalence of venous thromboembolism in patients with severe novel coronavirus pneumonia. $J$ Thromb Haemost, 18(6): 1421-24.

Dobesh, P. P. \& Trujillo, T. C. (2020). Coagulopathy, Venous Thromboembolism, and Anticoagulation in Patients with COVID-19. Pharmacoterapy, 40(11),01-53.

Eljilany, I. \& Elzouki, A. (2020). D-Dimer, Fibrinogen, and IL-6 in COVID-19 Patients with Suspected Venous Thromboembolism: A Narrative Review. Vascular Health and Risk Management, 16, 455-462.

Fauvel, C., Weizman, O., Trimaille, A., Mika, D., Pommier, T., Pace, N. .. Pezel, T. (2020). Pulmonary embolism in COVID-19 patients: a French multicentre cohort study. European Heart Journal, 41(32), 3058-3068.

Fu, Z., Zhuang, X., He, Y., Huang, H \& GUO, W. (2020). The diagnostic value of D-dimer with simplified Geneva score (SGS) pre-test in the diagnosis of pulmonary embolism (PE). Journal of Cardiothoracic Surgery, 15(176), 01-07.

Galvão, T. F., Pansani, T. S. A., Harrad, D. (2015). Principais itens para relatar Revisões sistemáticas e Meta-análises: A recomendação PRISMA. Epidemiol. Serv. Saúde, 24(02), 335-342.

Garcia-Olivé, I., Sintes, H., Radua, J., Capa, J.Á. \& Rosell, A. (2020). D-dimer in patients infected with COVID-19 and suspected pulmonary embolism. Respiratory Medicine, 169(106023), 01-03.

García-Ortega, A., Oscullo, G., Calvillo, P., MARTÍ-BONMATI, L., MENÉNDEZ, R. \& MARTÍNEZ-GARCIA, M.A. (2020). Incidence, risk factors, and thrombotic load of pulmonary embolism in patients hospitalized for COVID-19 infection. Journal of infection, 82(02), 261-69.

Gomes, L. M., Marchiori, E. \& Rodrigues, R. S. (2006). Trombose venosa profunda e suspeita de tromboembolismo pulmonar: avaliação simultânea por meio de angiotomografia pulmonar e venotomografia combinadas. Radiol Bras, 39(1), 19-26.

Haque, S., Jawed, A., Akhter, N., Dar, S. A., Khan, R. K., MandaL, M. Y. .. YahiD, M. (2020). Acetylsalicylic acid (Aspirin): a potent medicine for preventing COVID-19 deaths caused by thrombosis and pulmonary embolism. Eur Rev Med Pharmacol Sci, 24(18),9244-9245.

Klok, F. A., Mos. I. C. M., Nijekeuter. M., Righini, M., Perrier, A. \& GaL, L.G. (2008). Simplification of the Revised Geneva Score for Assessing Clinical Probability of Pulmonary Embolism. Arch intern med, 168(19), 2131-36.

Li, M., Gitarts, S., Nyabera, A., Kondaveeti, R., Hammudeh, Y. \& Gonzalez, C. (2020). Continuous Infusion Low-Dose Unfractionated Heparin for the Management of Hypercoagulability Associated With COVID-19. Journal of Pharmacy Practice, 20(10), 01-07.

Liao, S., Shao, S., Chen, Y., Chen, Y. \& PEndurado M. (2020) Incidence and mortality of pulmonary embolism in COVID-19: a systematic review and metaanalysis. Critical Care, 464(24), 01-05.

Lippi, G., Plebani, M. \& Henry, B. M. (2020). A trombocitopenia está associada a infecções graves por coronavírus 2019 (COVID-19): uma meta-análise. Clin Chim Acta, 506, 145-148.

Rossi, F. (2020). Tromboembolismo venoso em pacientes COVID-19. Jornal Vascular Brasileiro, 19, 01-05.

Lodigiani, C., Lapichino, G., Carenzo, L., Cecconi, M., Ferrazzi, P., Sebastian, T. ... Barco, S. (2020). Thrombosis Research,191,9-14.

Long, H., Nie, L., Xiang, X., Li, H., Zhang, X., Fu, X. ... Wu, Q. (2020). D-Dimer and Prothrombin Time Are the Significant Indicators of Severe COVID-19 and Poor Prognosis. BioMed Research International, 01-10.

Manolis, A. S., Manolis, T. A. \& Manolis, A. A. (2021). COVID-19 Infection: Viral Macro- and Micro-Vascular Coagulopathy and Thromboembolism/Prophylactic and Therapeutic Management. Journal of Cardiovascular Pharmacology and Therapeutics, 26(1), 12-24.

Marietta, M., Coluccio, V. \& Luppi, M. (2020). COVID-19, coagulopatia e tromboembolismo venoso: mais perguntas do que respostas. Estagiário Emerg Med, 12(8), 01-13.

Marinho, F. P., Loyola, I. S., Monteiro, I. O. F., Castro, T. M., Carvalho, M. G. S. ... Santos, G. B. (2021). Inter-relação entre COVID-19 e diabetes mellitus: uma revisão sistemática. Research, Society and Development, 10(2), 01-14.

Mendes, K. D. S., Silveira, R. C. C. P. \& Galvão, C. M. (2008). Revisão integrativa: método de pesquisa para a incorporação de evidências na saúde e na enfermagem. Texto Contexto Enfermagem, 17(4),758-64.

Melnyk, B. M. \& Fineout-Overholt, E. (2005). Making the case for evidence-based practice.In: Melnyk BM, Fineout-Overholt E. Evidence-based practice in nursing \& healthcare. A guide to best practice. Philadelphia: Lippincot Williams \& Wilkins, 3-24.

Mestre-Gómez, B., Lorente-Ramos, R. M., Rogado, J., Franco-Moreno, A., Obispo, B., Salazar-Chiriboga, D. ... Rivas, N. M. (2020). Incidence of pulmonary embolism in non-critically ill COVID-19 patients. Predicting factors for a challenging diagnosis. Journal of Thrombosis and Thrombolysis, 51(01), 40-46. 
Middeldorp, S., Coppens, M., Haaps, T. F. V., Foppen, M., Vlaar, A. P., Muller, M. C. A., Bouman, C. C. S., Beenen, F. F. M., Kootte, R. S., Heijmans, J., Smits, L. P., Bonta, P. \& Es, N. V. (2020). Incidência de tromboembolismo venoso em pacientes hospitalizados com COVID-19. J Thromb Haemost., 18 (08), 1995-2002.

Minno, A. D., Ambrosino, P., Calcaterra, I., Minno, M. N. D. D. (2020). COVID-19 and Venous Thromboembolism: A Meta-analysis of Literature Studies. Seminars in Thrombosis \& Hemostasis, 46(7), 763-771.

Nadkarni, G. N., Lala, A., Bagiella, E., Chang, H. L., Moreno, P. R. \& Pujadas, E. (2020). Anticoagulation, Bleeding, Mortality, and Pathology in Hospitalized Patients With COVID-19. Journal of the American college of Cardiology, 76 (16),1815-1826.

Nopp, S., Florian, K., Jilma, B., PabingeR, I. \& A. Y, C. (2020). Risk of venous thromboembolism in patients with COVID-19: A systematic review and meta-analysis. Res Pract Thromb Haemost, 4(07), 1178-1191.

Ooi, M. Y. X., Rajai, A., Patel, R., Gerova, N., Godhamgaokar, V. \& Liong, S. Y. (2020). Pulmonary thromboembolic disease in COVID-19 patients on CT pulmonary angiography - Prevalence, pattern of disease and relationship to D-dimer. Europen jornal of radiology,132(109336), 01-07.

Parks, A. L., Auerbach, A. D., Schinipper, J. L., Anstey, J. E., sterken, D. G., Hecht, T. E. H. \& Fang, M. C (2020). COVID-19 coagulopathy and thrombosis: Analysis of hospital protocols in response to the rapidly evolving pandemic. Trombosis Research, 196, 355-358.

Pavoni, V., Gianesello, L., Pazzi, M., Stera, C. \& Meconi, T. (2020). Venous thromboembolism and bleeding in critically ill COVID-19 patients treated with higher than standard low molecular weight heparin doses and aspirin: A call to action. Trombosis Research, 196,313-317.

Poulakou, G., Dimakakos, E., Kollias, A., Kyriakoulis, K. G., Rapti, V., Trontzas, I. ... Syrigos, K. N. (2021). Beneficial Effects of Intermediate Dosage of Anticoagulation Treatment on the Prognosis of Hospitalized COVID-19 Patients: The ETHRA Study. In vivo, 35, 653-661.

Rashidi, F., Barco, S., Kamangar, F., Movalled, K., Ghorbani, O., Ansarin, K. ... Ansarin, K. (2021). Incidence of symptomatic venous thromboembolism following hospitalization for coronavirus disease 2019: Prospective results from a multi-center study. Trombosis research, 198,135-38.

Rentsch, C. T., Beckman, J. A., Tomlinson, L., Gellad, W. F., Alcorn, C., Kidway-Khan, F. ... Freiberg, M. J. (2021). Early initiation of prophylactic anticoagulation for prevention of coronavirus disease 2019 mortality in patients admitted to hospital in the United States: cohort study. BMJ, $372(31), 01-13$.

Robert-Ebadi, H., Mostaguir, K., Hovens, M., Kare, M., Verschuren, F., Girard, P., Huisman, M. V. ... Gal, G. L. (2017). Acessing clinical probability of pulmonar embolism: prospective validation of the simplified Geneva score. T Tromb Haemost, 15(09), 1764-1769.

Rosovsky, R. P., Grodzin, C., Channick, R., Davis, G. A., Giri, J. S., Horowitz, J. ... Rosenfield, K. (2020). Diagnosis and Treatment of Pulmonary Embolism During the Coronavirus Disease 2019 Pandemic. Pulmonary and Cardiovascular: Special Features, 28(6),2590-2601.

Stessel, B., Vanvuchelen, C., Bruckers, L., Herbots, L., RamaekerS, D. \& Dubois, J. (2020). Impact of implementation of an individualised thromboprophylaxis protocol in critically ill ICU patients with COVID-19: A longitudinal controlled before-after study Trombosis research, $194,209-215$.

Smith, K., Krajeswki, K. C. \& Krajeswiki, M. P. (2020). Practical Considerations in Prevention and Treatment of Venous Thromboembolism in Hospitalized Patients with COVID-19. Am j health-syst pharm, 20(20), 01-07.

Spyropoulos, A. \& Weitz, J. I. (2020). Hospitalized COVID-19 patients and venous tromboembolism. Circulation, 142(2),129-132.

Taccone, F. S., Gevenois, P. A., Peluso, L., Pletchette, Z., Lheureux, O., Bresseur, A. ... Vicent, J. (2020). Higher Intensity Thromboprophylaxis Regimens and Pulmonary Embolism in Critically Ill Coronavirus Disease 2019 Patients. Crit Care Med, 48(11), 1087-1090.

Tang, N., Bai, H., Chen, X., Gong, J., Li, D. \& Sun, Z. (2020). Anticoagulant Treatment is associated with decreased mortality in severe coronavírus disease 2019 patients with coagulopathy. J trob Haemost, 18,1094-1099.

Trigonis, R. A., Holt, D. B., Yuan, R., Siddiqui, A. A., Craft, M. K., Khan, B. A. .. Rahman, O. (2020). Incidence of Venous Thromboembolism in Critically Ill Coronavirus Disease 2019 Patients Receiving Prophylactic Anticoagulation. Critical Care medicine, 98(07), 805-808.

Valle, C., Bonafinni, P. A., Corso, M. D., Vacca, G., Gianatti, A. \& Sironi, S. (2020). Association between pulmonary embolism and COVID-19 severe pneumonia: Experience from two centers in the core of the infection Italian peak. European Journal of radiology,137(109613),01-08.

Vernuccio, F., Lombardo, F. P., Cannella, R., Panzuto, F., giambeluca, D., Arzanauskaite, M. ... Cabassa, P. Thromboembolic complications of COVID-19: the combined effect of a pro-coagulant pattern and an endothelial thrombo-inflammatory syndrome. Clinical radiology 2020; 75(11):804-10.

Yang, L., Liu, S., Liu, J., Zhang, Z., Wan, X., Huang, B., Chen, Y. \& Zhang, Y. (2020). COVID-19: immunopathogenesis and Immunotherapeutics. Sig Transduct Target Ther, 128(5), 01-08.

Zhai, Z., Li, C., Chen, Y., Gerotziafas, G., Zhang, Z., Whan, J., Liu, P., Elalamy, I. \& Whang, C. (2020). Prevention and Treatment of Venous Thromboembolism Associated with Coronavirus Disease 2019 Infection: A Consensus Statement before Guidelines. J Thromb Haemost., 120(06), 937-48. 